\title{
Parafoil Control Using Payload Weight Shift
}

\author{
Michael Ward, Sean Culpepper,, \pm and Mark Costello \\ Georgia Institute of Technology, Atlanta, Georgia 30332 \\ DOI: $10.2514 / 1 . C 032251$
}

\begin{abstract}
Autonomous guided airdrop systems based on steerable ram-air parafoils rely on differential deflection of the canopy trailing edge for lateral control with very limited longitudinal control. This work explores an alternative method that achieves both lateral and longitudinal control by shifting the center of gravity of the payload relative to the canopy rigging attachments. A multibody simulation model is required to capture the complex motion associated with the flexible nature of the parafoil, payload, and rigging system and simulate the effects of both longitudinal and lateral shifting of the center of gravity. Simulation results show that longitudinal weight shift can be an effective means of providing airspeed and glide slope control, and lateral weight shift can be an effective means of providing turn rate control. Results demonstrate that, for both lateral and longitudinal control, spreading the attachment points of the rigging to the payload will cause the aircraft to be more sensitive to shifts in the payload center of gravity. It was also found that, for both lateral and longitudinal control, decreasing the vertical distance from the rigging attachments to the center of gravity on the payload causes the aircraft to be more sensitive to shifts in the payload center of gravity. Finally, dynamic results demonstrate that the forces and moments required to induce a weight shift in the payload will often cause an initial response in the opposite direction of the final steady-state response.
\end{abstract}

\section{Nomenclature}

$A, B, C$

$b$

$c$

$F_{X}, F_{Y}, F_{Z}$

$\mathbf{I}_{B}, \mathbf{J}_{B}, \mathbf{K}_{B}$

$\mathbf{I}_{C}, \mathbf{J}_{C}, \mathbf{K}_{C}$

$m$

$M_{X}, M_{Y}, M_{Z}$

$P, Q, R$

$p, q, r$

$R$

$S$

$\tilde{u}, \tilde{v}, \tilde{w}$

$\tilde{V}$

$\alpha$

$\beta$

$\Delta b$

$\Delta c$

$\Delta h$

$=$

$=$

$=$

$=$

$=$

mass velocity

$=$ canopy arc radius

$=$ canopy reference area respect to air

$=$ angle of attack

$=$ angle of sideslip

= canopy pitch angle payload on payload basis vectors for canopy reference frame

= moment measure numbers in canopy frame

$=$ Lamb's coefficients for apparent inertia

= canopy frame components of canopy angular

= canopy frame components of velocity of aerodynamic center with respect to air

$=$ velocity of canopy aerodynamic center with

$=$ lateral separation of rigging attachments on

$=$ longitudinal separation of rigging attachments

$=$ vertical separation of rigging attachments from payload center of gravity

\section{Introduction}

A IRDROP systems provide a unique capability of delivering large payloads to undeveloped and inaccessible locations. Beginning in the 1990s, autonomous guided airdrop systems based on steerable ram-air parafoils were developed with the goal of improving the precision and accuracy of air-dropped payload delivery [1]. Parachutes are normally not thought of as aircraft, but a parafoil-and-payload system is able to glide to penetrate winds or reach targets from significant release offsets, steer to track a desired heading, and (with the addition of propulsion) takeoff and climb without assistance. For these reasons, the combined system of a parafoil and payload is referred to throughout this work as a parafoiland-payload aircraft. Research and development work on guided airdrop systems has focused primarily on improving the guidance algorithm [2-8]. The control mechanism used by current autonomous systems is based solely on deflection of the trailing edge, also referred to as brake deflection. Asymmetric deflection of the trailing-edge brakes provides effective lateral control. Symmetric deflection of the trailing brakes provides an increase in both lift and drag, which results in a decrease in airspeed. However, the ratio of lift to drag, and hence the glide angle, is relatively unchanged until the canopy nears stall. The current work examines an alternative control mechanism based on shifting the center of gravity of the payload relative to the rigging attachment points.

Weight shifting as a control mechanism is well established for certain classes of light aircraft. Weight-shift-controlled microlight aircraft, known as "trike" or "flexwing," rely strictly on pitching or rolling moments induced through a control bar that shifts the center of gravity [9]. Foot-launch hang gliders have always relied heavily on weight shift for control [10-13]. Pilots of high-performance parafoils referred to as paragliders use weight shift in conjunction with trailingedge brake deflection for efficient lateral control [14], and small remote-control-powered parafoils often use lateral weight shift as the sole means for lateral control.

Weight shift offers a number of interesting advantages compared to conventional control methods for parafoils, such as allowing all of the control mechanisms to be isolated in the payload and eliminating the need for canopy deformation. On the other hand, large displacements of the center of gravity relative to the rigging attachments are required for weight shift control, which requires very powerful actuators. This makes weight shift control a more appropriate control mechanism for smaller systems where actuator weight and cost are less of a concern. Lateral weight shift can be used to induce a turn, while longitudinal weight shift can be used to induce a change in the trim angle of attack that produces both a change in airspeed and glide angle. The current work presents an in-depth examination of the effects of both 
longitudinal and lateral weight shifts on the flight characteristics of a parafoil-and-payload system. Both the steady-state and dynamic responses to longitudinal and lateral weight shifts are examined, and the effect of the payload rigging attachment geometry on the effectiveness of the weight shift control mechanism is studied.

\section{Parafoil and Payload Model}

On a parafoil-and-payload aircraft, weight shift control works by shifting the center of mass of the payload relative to the rigging attachment points. This alters the forces on the rigging lines, and hence on the parafoil canopy. The altered forces on the parafoil canopy result in a change in the canopy's orientation with respect to the air, which in turn alters the flight condition of the vehicle. In the case of longitudinal weight shift, the effect is to alter the pitch angle of the canopy, which alters the trim angle of attack, and in turn the airspeed and glide ratios. In the case of lateral weight shift, a bank angle is induced in the canopy that produces a turn. The connection between the payload and canopy is flexible by nature, and the effect of weight shift on a payload-and-parafoil combination must necessarily depend on the geometry of the rigging that connects them. This means that any realistic attempt at simulating the effect of weight shift on a parafoil-and-payload aircraft must model the aircraft as a flexible, multibody system.

A common approach to modeling parafoil-and-payload aircraft is to represent the entire system as a single rigid body with six degrees of freedom [15-17]. This approach is an extremely efficient method for evaluating guidance, navigation, and control techniques, but it neglects the relative motion of the payload and canopy. A common approach to capture the relative motion is to model the payload and canopy as two separate rigid bodies and to approximate the flexible rigging geometry by either a single flexible joint [18-21] or a carefully chosen set of flexible joints and rigid links [22-23]. This is the approach used for the current work because it allows the effect of the key rigging geometry variables on weight shift to be explored while retaining the computational efficiency and simplicity of rigidbody modeling techniques.

Forming the dynamic equations of motion for a multibody dynamic system is much more complex owing to the existence of internal constraint forces and moments that connect the system together. The method used here treats each rigid body of the aircraft as a general rigid body with six degrees of freedom. All constraint forces and moments that arise from system connections (hinge joint, sliders, etc.) are treated as external loads that are equal and opposite on connecting bodies. For the $i$ th rigid body of the system, the dynamic equations of motion can be written in the following affine form:

$$
\dot{X}_{i}=F_{i}+G_{i} \mathbf{U}
$$

In the preceding equation, $X_{i}$ is the state vector of the $i$ th rigid body of the system, $F_{i}$ represents the unconstrained dynamic equations of the $i$ th rigid body, and $G_{i} \mathbf{U}$ represents the contributions of the connection constraint forces and moments to the dynamic equations. The vector $\mathbf{U}$ contains force and moment constraints computed using a feedback linearization technique to enforce the joint constraints and ensure that the multibody systems remains "glued" together. A complete derivation of this multibody modeling technique using a "glue code controller" can be found in [24,25].

For forming flight dynamic equations of motion, the method has the advantage of leveraging rigid six-degree-of-freedom dynamic modeling that is well known to the flight dynamic community. A multibody dynamic simulation can be constructed by the simple addition of appropriate constraint forces and moments to the rigid six-degree-of-freedom model along with the addition of a system glue code controller, which is a function of the connectivity of the multibody system.

Figure 1 shows a schematic of a parafoil-and-payload system. The parafoil and payload are modeled as separate rigid bodies. The effect of the rigging lines are modeled by using rigid links of negligible mass and inertia to connect the canopy to the payload.

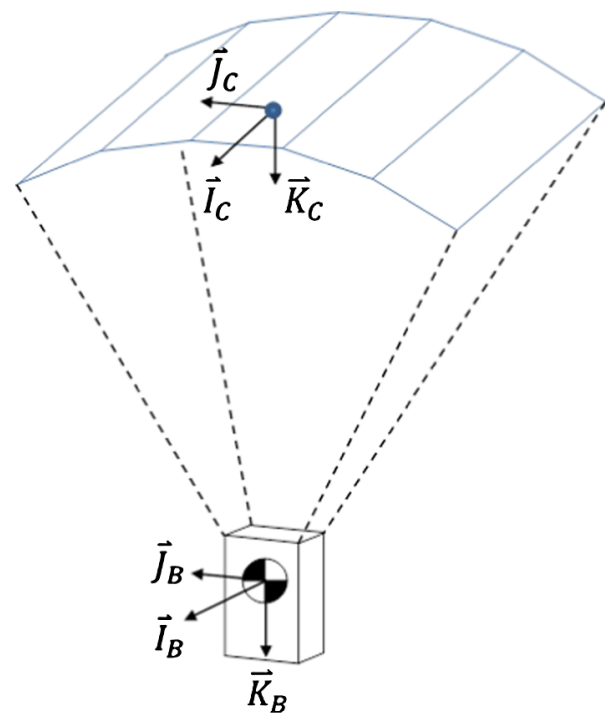

Fig. 1 Schematic of simulation model.

Beginning with the canopy, the origin of the canopy coordinate frame is placed at the aerodynamic center of the canopy. The coordinate system is aligned with the canopy such that the $x$ axis points out the front of the canopy and is parallel with the bottom surface of the canopy. Parafoil canopies are constructed entirely of fabric with no rigid members and are typically less than $1 \%$ of the weight of the payload, so the weight of the canopy is neglected. The aerodynamic velocity components of the canopy expressed in the canopy reference frame are denoted with tildes $(\tilde{u}, \tilde{v}$, and $\tilde{w})$ so that the airspeed, angle of attack, and angle of sideslip are given by

$$
\tilde{V}=\sqrt{\tilde{u}^{2}+\tilde{v}^{2}+\tilde{w}^{2}}, \quad \alpha=\tan ^{-1} \frac{\tilde{w}}{\tilde{u}}, \quad \beta=\sin ^{-1} \frac{\tilde{v}}{\tilde{V}}
$$

The aerodynamic forces on the canopy are computed as functions of these aerodynamic quantities:

$$
\left\{\begin{array}{l}
F_{X, \text { canopy }} \\
F_{Y, \text { canopy }} \\
F_{Z, \text { canopy }}
\end{array}\right\}=\frac{1}{2} \rho \tilde{V}^{2} S\left[T_{a}\right]\left\{\begin{array}{c}
-\left(C_{D 0}+C_{D A 2} \alpha^{2}\right) \\
C_{Y \beta} \beta+b / 2 \tilde{V}\left(C_{Y p} p+C_{Y r} r\right) \\
-\left(C_{L 0}+C_{L A} \alpha\right)
\end{array}\right\}
$$

where $\left[T_{a}\right]$ is a $y$-axis rotation by the angle of attack. The aerodynamic moments are functions of the angular rates and the sideslip angle:

$$
\left\{\begin{array}{l}
M_{X, \text { canopy }} \\
M_{Y, \text { canopy }} \\
M_{Z, \text { canopy }}
\end{array}\right\}=\frac{1}{2} \rho \tilde{V}^{2} S\left\{\begin{array}{c}
b\left(b / 2 \tilde{V}\left(C_{l p} p+C_{l r} r\right)+C_{l \beta} \beta\right) \\
c^{2} / 2 \tilde{V} C_{m q} q \\
b\left(b / 2 \tilde{V}\left(C_{n p} p+C_{n r} r\right)+C_{n \beta} \beta\right)
\end{array}\right\}
$$

The lateral dynamics are fully coupled so that a change in sideslip, roll rate, or yaw rate will produce a change in the side force, roll moment, and yaw moment. It is possible to obtain reasonable representations of the canopy aerodynamics when using conventional control inputs that modify the aerodynamic forces and moments directly. However, weight shift produces only a change in the orientation of the canopy without a direct modification of the aerodynamic forces and moments. This means that it is critical to model the coupling between the roll, yaw, and sideslip dynamics to reproduce the effect of lateral weight shift.

Moving on to the payload, the origin of the reference frame associated with the payload is placed at the center of mass of the payload that, since gravitational forces on the canopy are neglected, is also the center of gravity of the entire system. The orientation of this coordinate system is such that the $x$ axis points forward and is parallel 
to a longitudinal line drawn along the points where the canopy is attached to the payload. The aerodynamic area of the payload is very small compared to the canopy (typically less than $0.5 \%$ ), and the aerodynamic forces on the payload are neglected so that the only force on the payload is gravity. For the example systems simulated in this work, this assumption results in an alteration of the trim pitch angles by no more than $0.4 \mathrm{deg}$, and it results in an alteration of the time to half of the pitch oscillations of no more than $0.1 \mathrm{~s}$.

\section{A. Longitudinal Multibody Model}

In addition to the canopy and payload, two rigid links with negligible mass and inertia are used in the simulation to connect the canopy to the payload. Laterally, the links are attached to the centerline of both the canopy and payload. The top of the links are attached to the front and back of the canopy. The bottom of the links are attached to the payload with longitudinal separation distance $\Delta c$. This results in a total of four bodies connected by four pin joints in the simulation. Every joint allows free rotation of the bodies but constrains translation.

For the longitudinal model, the dynamics are simple enough to construct a simple static model to compute trim in an extremely efficient manner. The unknown variables include the flight conditions (angle of attack and airspeed), canopy pitch angle, and the payload pitch angle. The constraints are the fixed line lengths, chord length, and rigging attachment geometry as well as force and moment equilibrium over the entire system.

The algorithm uses an iterative process beginning with an initial guess for the canopy and payload pitch angles, calculating the rigging geometry based on the geometry constraints, and then calculating the forces and moments on the system. The residuals are the inertial frame components of the forces and moments on the system, and they

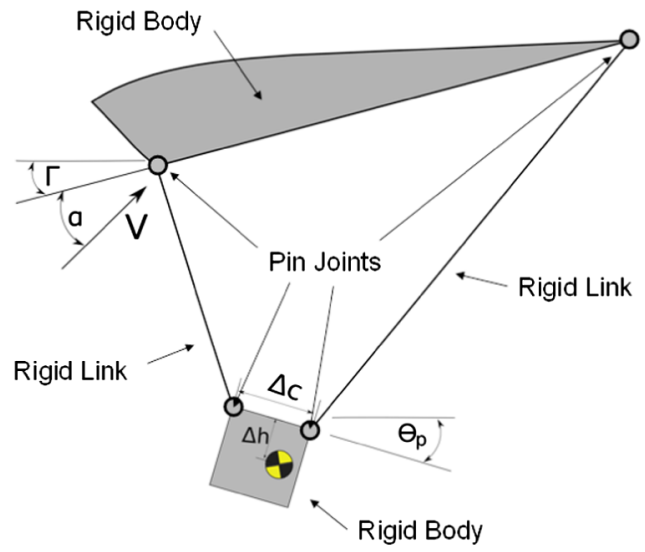

Fig. 2 Parafoil payload schematic for longitudinal weight shift simulation.

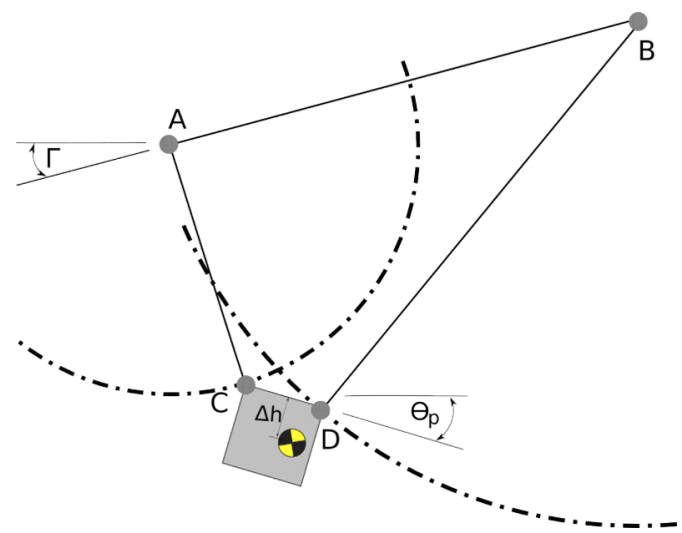

Fig. 3 Four-bar linkage geometry. are described next. The equations can be solved numerically [26] to find the trim flight conditions and canopy and payload pitch angles corresponding to a given c.g. location. The geometry of the longitudinal simulation model is shown in Fig. 2.

The rigging geometry is modeled as a four-bar linkage consisting of massless rigid bars of constant length connected tip to tail by hinges, resulting in a closed geometry. The lengths of all the bars are known, and the canopy and payload pitch angles are states. The angles of the front and rear lines are unknown and must be computed. The problem can be visualized by drawing circles about the tip and tail of the canopy that have radii equal to the front and rear line lengths, respectively, as seen in Fig. 3 .

The solution can then be found as the points where the distance between the circles equals $\Delta c$ and have an angle that matches the given payload angle. Solving for each hinge point was accomplished using an iterative approach. Point A was assumed to be at the origin, and point $\mathrm{B}$ was calculated directly from the current guess for the canopy pitch angle and the known chord length:

$$
B_{x}=c \cos \Gamma, \quad B_{y}=c \sin \Gamma
$$

Point $\mathrm{D}$ is placed to relative to point $\mathrm{C}$,

$$
D_{x}=\Delta c \cos \theta_{p}+C_{x}, \quad D_{y}=\Delta c \sin \theta_{p}+C_{y}
$$

and point $\mathrm{C}$ is found by an iterative procedure to satisfy the line length constraints:

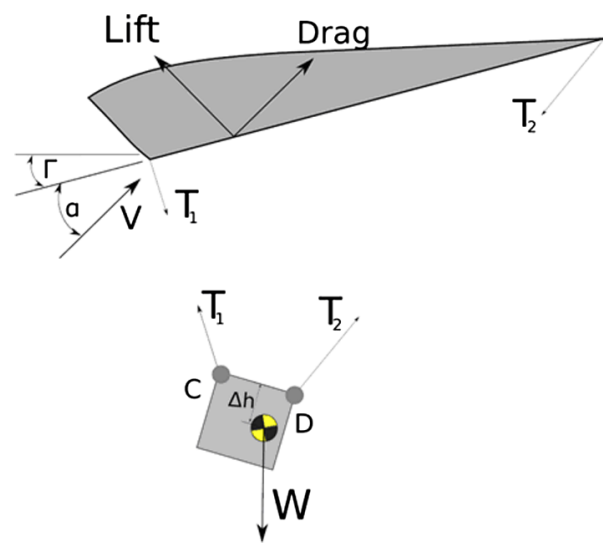

Fig. 4 Canopy and payload free body diagrams.

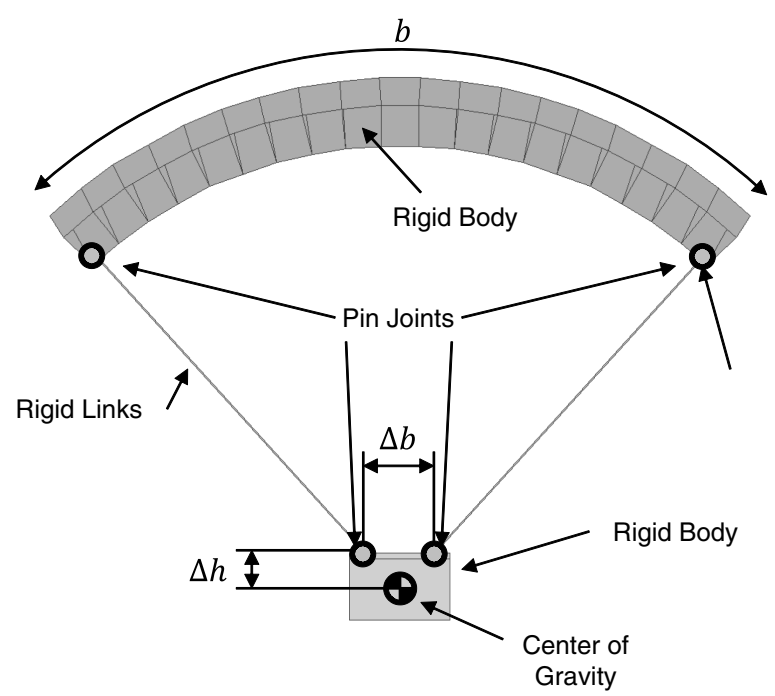

Fig. 5 Parafoil payload schematic for lateral weight shift simulation. 

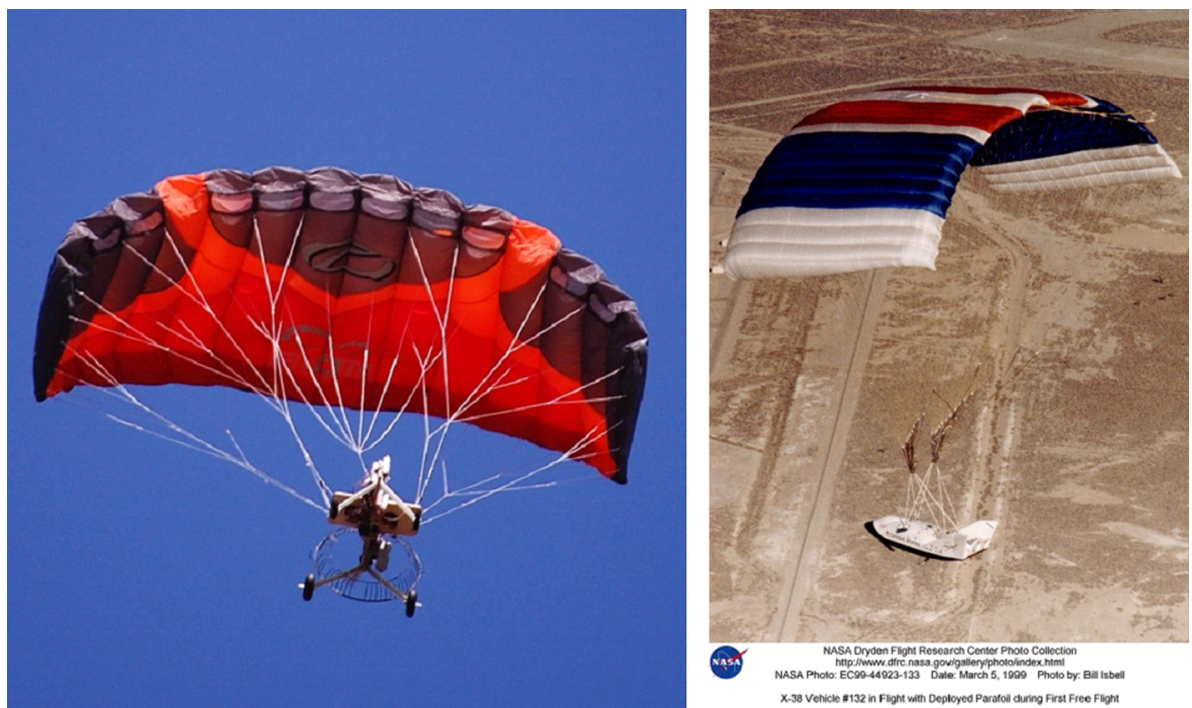

Fig. 6 Example parafoil systems: GT Imp (left) and X-38 (right).

$$
\begin{gathered}
l_{f l}^{2}=C_{x}^{2}+C_{y}^{2} \\
l_{t l}^{2}=\left(D_{x}-B_{x}\right)^{2}+\left(D_{y}-B_{\mathrm{y}}\right)^{2}
\end{gathered}
$$

To develop the force and moment balance equations, the canopy and payload are considered separately, as shown in Fig. 4.

For the canopy, the sum of forces and the moments about the aerodynamic center should be zero:

$$
\begin{gathered}
\mathbf{T}_{1}+\mathbf{T}_{2}+\mathbf{L}+\mathbf{D}=0 \\
\mathbf{r}_{\mathrm{ac} \rightarrow A} \times \mathbf{T}_{1}+\mathbf{r}_{\mathrm{ac} \rightarrow B} \times \mathbf{T}_{2}=0
\end{gathered}
$$

For the payload, the sum of forces and moments about the center of gravity should be zero:

$$
\begin{gathered}
-\mathbf{T}_{1}-\mathbf{T}_{2}+\mathbf{W}=0 \\
\mathbf{r}_{\mathrm{cg} \rightarrow C} \times \mathbf{T}_{1}+\mathbf{r}_{\mathrm{cg} \rightarrow D} \times \mathbf{T}_{2}=0
\end{gathered}
$$

The lift and drag forces are functions of angle of attack and airspeed and are calculated based on the current states for the flight condition. Equations (9-12) yield six equations when written in inertial frame components. The direction of the tension forces $T_{1}$ and $T_{2}$ are constrained by the rigging geometry, so the magnitudes of these forces are the only unknowns. This leaves four equations to be used as residuals in the numerical solution procedure to solve for the four unknown states of the system (angle of attack, airspeed, canopy pitch, and payload pitch).

\section{B. Lateral Multibody Mode}

The lateral simulation model also makes use of two rigid links with negligible mass and inertia to connect the canopy to the payload. The tops of the links are attached to the left and right wingtips of the canopy, longitudinally aligned with the canopy aerodynamic center. The bottoms of the links are attached to the payload with lateral separation distance $\Delta b$, longitudinally aligned with the payload center of mass. As in the longitudinal model, this results in a total of four bodies connected by four pin joints in the simulation. Every joint allows free rotation of the bodies but constrains translation. The geometry of the lateral simulation model is shown in Fig. 5.

The lateral dynamics of a parafoil-and-payload aircraft are significantly more complicated than the longitudinal dynamics. The roll, yaw, and sideslip motions are heavily coupled even at small turn rates; and at large turn rates, the lateral and longitudinal dynamics also become heavily coupled. This means that a simple static model of the parafoil-and-payload system cannot be used for lateral trim calculations in the manner used to perform the aforementioned longitudinal trim analysis. Instead, a dynamic simulation model is employed. To determine the effect of lateral weight shift, the payload center of gravity is shifted in the simulation model and a full, nonlinear dynamic model is propagated forward in time until a steady turn rate has been achieved.

\section{Example System}

The mass, geometry, and aerodynamic parameters for the simulation model were set to match two parafoil-and-payload systems: one very small and one very large. These systems were chosen to examine the effect of scale on payload weight shift as a control mechanism. The small system is called the GT-Imp and was flight tested by Ward et al. at the Georgia Institute of Technology [27]. The second system was flight tested by NASA for the X-38 program [28]. Both systems are shown in flight in Fig. 6. The mass and geometry parameters for the canopy and payload are given in

\begin{tabular}{|c|c|c|}
\hline Parameter & GT Imp & $\mathrm{X}-38$ \\
\hline \multicolumn{3}{|c|}{ Canopy } \\
\hline$S, \mathrm{~m}^{2}$ & 2.1 & 508 \\
\hline$b, \mathrm{~m}$ & 2.4 & 36.6 \\
\hline$c, \mathrm{~m}$ & 0.88 & 13.7 \\
\hline$R, \mathrm{~m}$ & 1.7 & 22.0 \\
\hline$m, \mathrm{~kg}$ & 0.2 & 50 \\
\hline$I_{x x}, \mathrm{~kg} \cdot \mathrm{m}^{2}$ & 0.6 & 35,000 \\
\hline$I_{y y}, \mathrm{~kg} \cdot \mathrm{m}^{2}$ & 0.05 & 3,200 \\
\hline$I_{z z}, \mathrm{~kg} \cdot \mathrm{m}^{2}$ & 0.7 & 40,000 \\
\hline$A, \mathrm{~kg}$ & 0.05 & 120 \\
\hline$B, \mathrm{~kg}$ & 0.35 & 1,300 \\
\hline$C, \mathrm{~kg}$ & 1.85 & 4,100 \\
\hline$P, \mathrm{~kg} \cdot \mathrm{m}^{2}$ & 0.07 & 33,000 \\
\hline$Q, \mathrm{~kg} \cdot \mathrm{m}^{2}$ & 0.06 & 42,000 \\
\hline$R, \mathrm{~kg} \cdot \mathrm{m}^{2}$ & 0.046 & 35,000 \\
\hline \multicolumn{3}{|c|}{ Payload } \\
\hline$m, \mathrm{~kg}$ & 3.7 & 6,180 \\
\hline$I_{x x}=I_{y y}=I_{z z}, \mathrm{~kg} \cdot \mathrm{m}^{2}$ & 1.6 & 25,000 \\
\hline
\end{tabular}
Table 1, and the aerodynamic parameters are given in Table 2 . The longitudinal aerodynamic parameters were set to match the flighttest data $[27,28]$, while the lateral aerodynamic parameters were determined using analytical expressions derived by Jann [29].

Table 1 Mass and geometry parameters 
Table 2 Aerodynamic parameters

\begin{tabular}{ccc}
\hline \hline Parameter & GT Imp & $\mathrm{X}-38$ \\
\hline$C_{L 0}$ & 0 & 0.4 \\
$C_{L A}$ & 3.56 & 5 \\
$C_{L A 3}$ & -28 & 0 \\
$C_{D 0}$ & 0.074 & 0.3 \\
$C_{D A 2}$ & 1.12 & 3 \\
$C_{m q}$ & 0 & 0 \\
$C_{Y \beta}$ & -0.48 & -1.08 \\
$C_{Y p}$ & 0.40 & 0.67 \\
$C_{Y r}$ & -0.05 & -0.17 \\
$C_{l \beta}$ & 0.20 & 0.34 \\
$C_{l p}$ & -0.29 & -0.34 \\
$C_{l r}$ & 0.036 & 0.08 \\
$C_{n \beta}$ & 0.005 & 0.015 \\
$C_{n p}$ & -0.007 & -0.015 \\
$C_{n r}$ & -0.048 & -0.11 \\
\hline \hline
\end{tabular}

\section{Longitudinal Weight Shift}

The purpose of longitudinal weight shift is to alter the trim pitch angle of the parafoil canopy. This produces a change in the angle of attack, which in turn results in a change in the airspeed and the glide ratio. The relationship between angle of attack, airspeed, and glide ratio will of course depend on the aerodynamic properties of the canopy. However, the effect of weight shift on canopy pitch angle depends only on the geometry of the parafoil-and-payload configuration. This means that it is possible to determine a relationship between pitch angle and flight condition, considering only the aerodynamics of the parafoil canopy, and it is possible to determine the relationship between weight shift and canopy pitch angle without assuming any aerodynamic properties. The glide ratio and airspeed vs pitch angle behavior for the two example simulation models are shown in Fig. 7 .
Figure 8 presents a visualization of the manner in which the system geometry changes with various weight shift inputs. In the center diagram, the center of gravity is at the nominal location, the payload pitch angle is zero, and the canopy pitch angle is at the nominal value. In the diagram on the left, the center of gravity is moved aft. As the center of gravity is moved aft, the payload and canopy pitch nose up. Due to the rigging geometry, the range of canopy pitch angle variation is much smaller than the range of payload pitch angle variation. The diagram on the right shows a forward shift of the center of gravity, which causes both the canopy and payload to pitch nose down.

The effect of longitudinal weight shift is to produce a nonlinear change in payload pitch angle that, through the rigging lines, induces a change in the canopy pitch angle. The sensitivity to weight shift is greatest near the nominal geometry when the payload pitch angle is small. As the magnitude of the payload pitch angle becomes large, the effect of weight shift is diminished. Eventually, a limit is reached where increasing weight shift produces no further change in the payload pitch angle and canopy pitch angle.

Figure 9 shows the steady-state canopy pitch angle resulting from longitudinal weight shift for both example systems. The longitudinal shift of the center of gravity is normalized by the canopy chord. The trim pitch angle for both systems is set to $-12 \mathrm{deg}$. For the X-38 canopy, the average rigging line length is $22 \mathrm{~m}$, the chord length is $13.7 \mathrm{~m}$, and the ratio of the rigging line length to the chord is 1.6 . For the GT-Imp, the average rigging length is $1.7 \mathrm{~m}$, the chord length is $0.88 \mathrm{~m}$, and the ratio of the line length to the chord is 1.93 . This difference in the rigging geometry between the two systems results in a slightly different response to longitudinal weight shift. If the rigging of the GT-Imp is shortened to $1.4 \mathrm{~m}$, yielding the same line length to chord ratio as the X-38, the steady-state canopy pitch angle response of the two systems to nondimensional weight shift is identical. The trim pitch angles of the canopy and payload depend only on the geometry of the rigging arrangement. If the shape of the rigging geometry of two systems is the same, then the trim pitch angle response to nondimensional weight shift will be the same. This
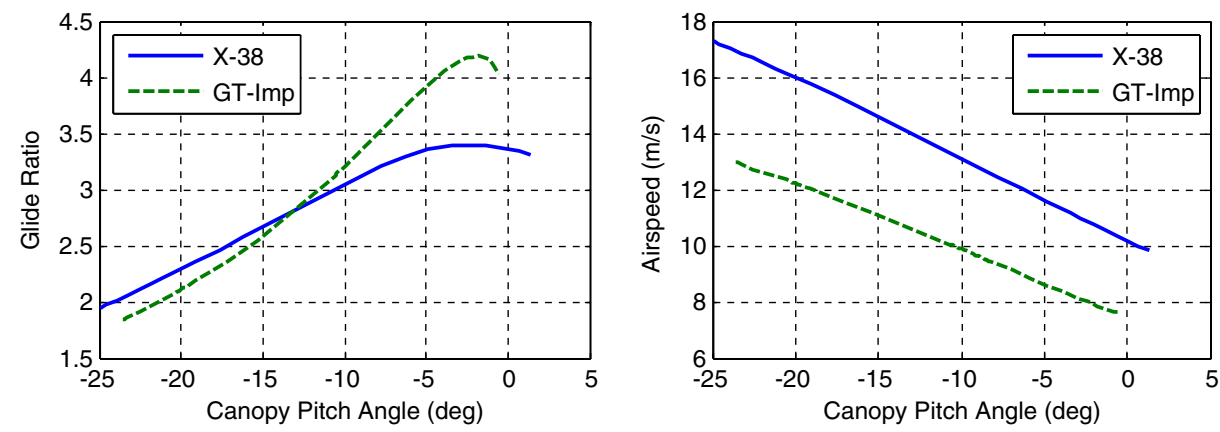

Fig. 7 Glide ratio and airspeed vs canopy pitch angle for example systems.
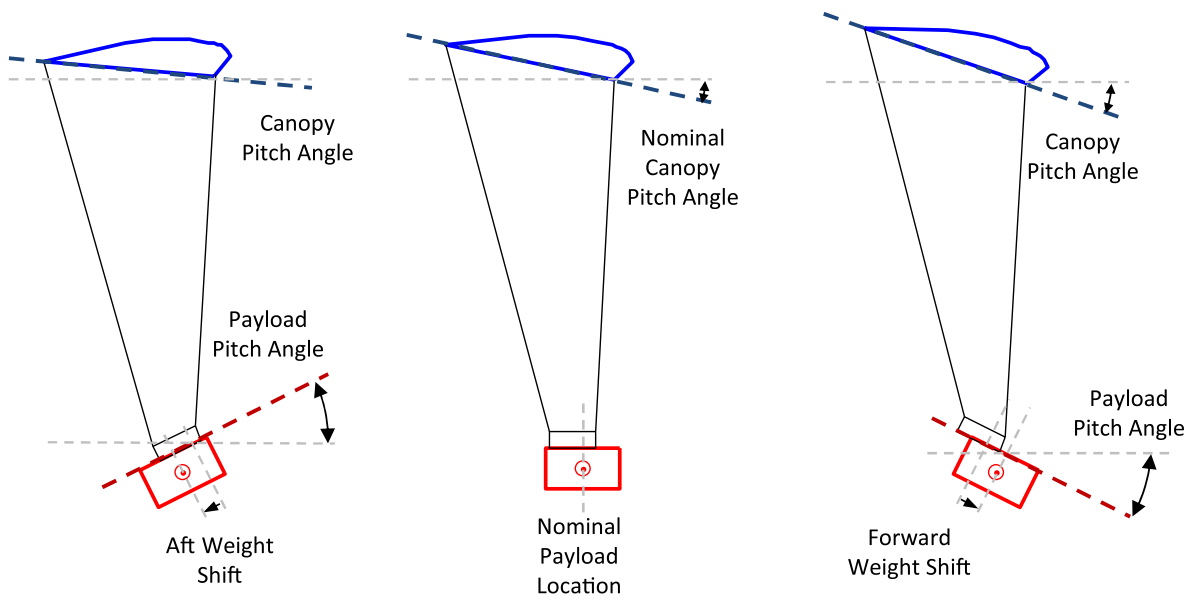

Fig. 8 Visualization of longitudinal weight shift. 


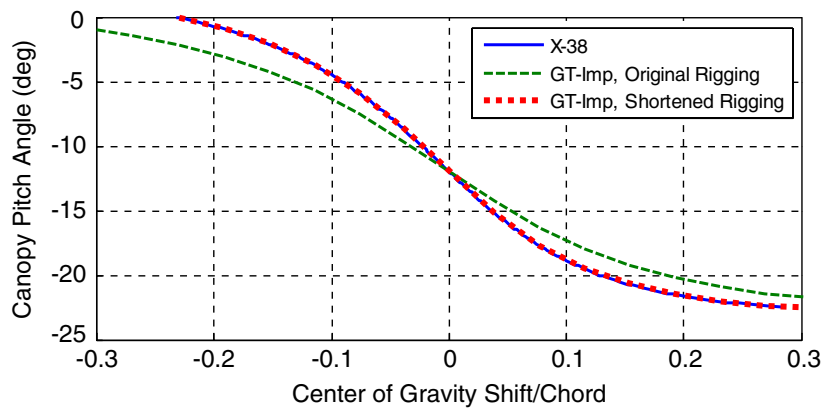

Fig. 9 Steady-state canopy pitch angle vs weight shift for example systems.

means that, by exploring the effectiveness of longitudinal weight shift in terms of nondimensional parameters, the results will be applicable to systems of any scale.

The average length of the rigging lines is normally set to ensure desirable flight dynamics of the parafoil-and-payload aircraft [30]. The geometry of the rigging attachments to the payload is a much more flexible design variable. The longitudinal separation of the rigging attachments ( $\Delta c$ in Fig. 2) is normally zero to prevent movement of the payload center of gravity from influencing the canopy trim angle. To implement weight shift control, the rigging attachments must be separated longitudinally to enable longitudinal weight shift to influence the trim pitch angle of the canopy. The dependence of the effectiveness of the weight shift control mechanism on the geometry of the rigging attachment points on the payload is explored by varying both the longitudinal separation of the attachment points and the vertical distance between the attachments and the center of gravity. A completely rigid representation of the system cannot model this behavior, so the multibody representation is essential.

Simulation results presented in Figs. 10-12 examine how the effect of longitudinal weight shift changes when varying the vertical distance of the center of gravity below the rigging attachments $(\Delta h$ in Fig. 2). The longitudinal weight shift is normalized by the canopy chord $c$, and the vertical distance is normalized by the average rigging

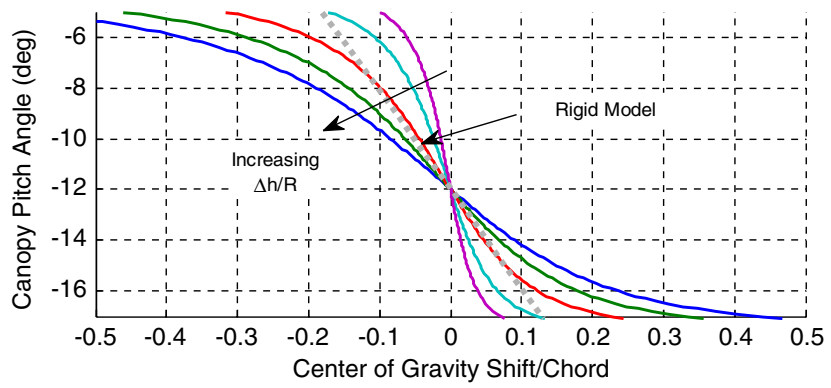

Fig. 10 Canopy pitch angle vs weight shift with varying heights of rigging attachments.

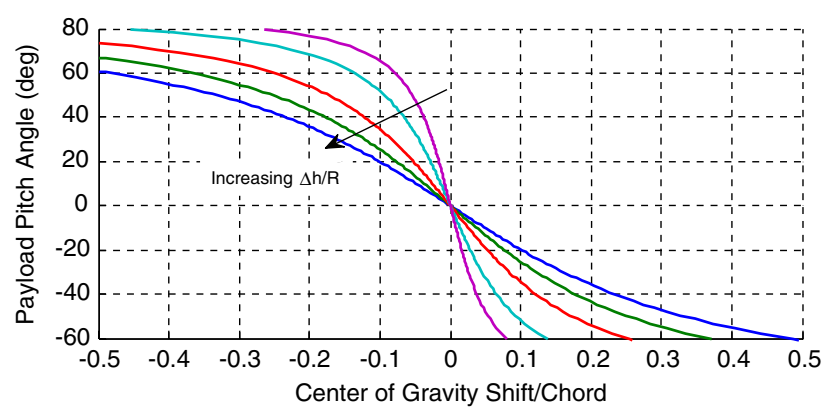

Fig. 11 Payload pitch angle vs weight shift with varying heights of rigging attachments.

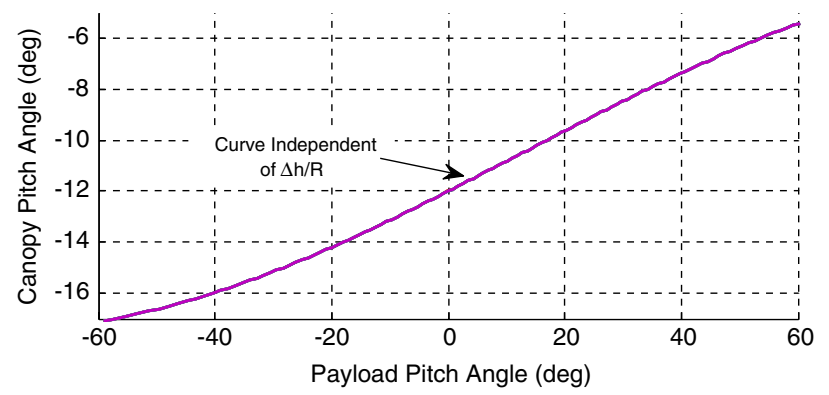

Fig. 12 Canopy pitch angle vs payload pitch angle with varying heights of rigging attachments.

line length. The c.g. shift was varied by $50 \%$ of the canopy chord length forward and aft of the nominal position, with the vertical distance values of $2.5,5,10,15$, and $20 \%$ of the average rigging line length. The longitudinal separation of the rigging attachment points was set to $10 \%$ of the canopy chord for all of these simulations.

In Fig. 10, in addition to the simulation results from the static trim analysis, the effect of weight shift on canopy pitch angle produced from a simulation model representing the entire parafoil-and-payload aircraft as a single rigid body is presented. In this rigid model, the relationship between weight shift and pitch angle is linear. The rigid model cannot predict the bounds on the range of control of pitch angle, and it cannot predict the effect of varying the rigging geometry. This is a clear example of the necessity of a multibody representation; a single rigid-body representation of the parafoil and payload cannot be used to produce realistic simulations of the effect of weight shift.

Note from Fig. 12 that the variation in the vertical distance of the center of gravity from the attachment points has no effect on the relationship between payload pitch angle and canopy pitch angle. The vertical distance only affects the amount of c.g. shift required to induce a given pitch angle in the payload. If the center of gravity is close to the attachment points, only a small shift in the center of gravity is required to produce a large change in the payload pitch angle. As the vertical distance is increased, a larger shift in the center of gravity is required to produce the same change in pitch angle. Because the relationship between payload pitch and canopy pitch is unchanged, the variation of the vertical distance does not change the range of control of canopy pitch angle.

Simulation results presented in Figs. 13-15 examine how the effect of longitudinal weight shift changes when varying the longitudinal separation of the rigging attachments ( $\Delta c$ in Fig. 2). Both the longitudinal weight shift and the rigging attachment separation are normalized by the canopy chord. The center of gravity was varied forward and aft of the nominal position, with the distance between the rigging attachments set to $1.25,2.5,5,7.5,10$, and $15 \%$ of the canopy chord. The vertical distance of the center of gravity from the rigging attachments was set to $10 \%$ of the average rigging line length for all of these simulations.

Note from Fig. 14 that the variation in the separation of the rigging attachment points has almost no effect on the relationship between weight shift payload pitch angle. There is a small variation in pitch angle, on the order of $5 \%$ for large values of weight shift, but the

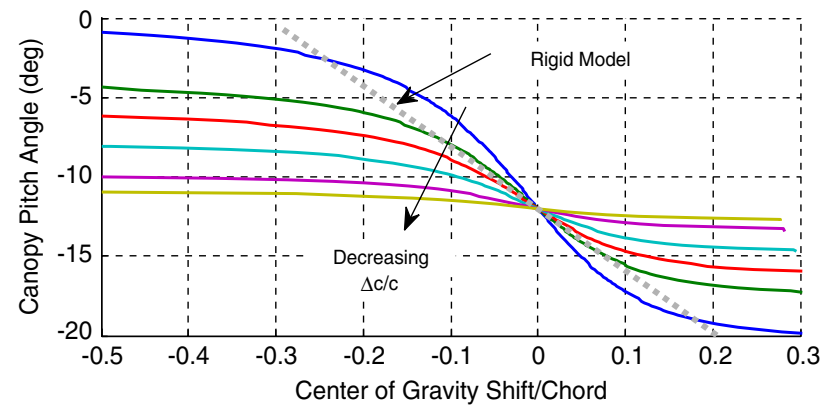

Fig. 13 Canopy pitch angle vs weight shift with separation of rigging attachments. 


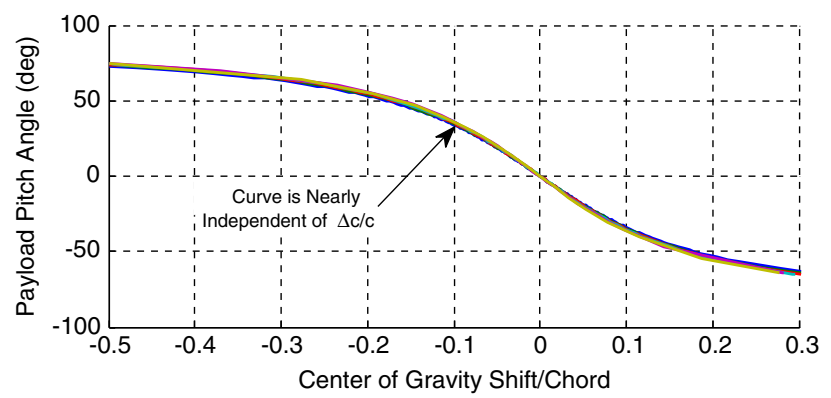

Fig. 14 Payload pitch angle vs weight shift with separation of rigging attachments.

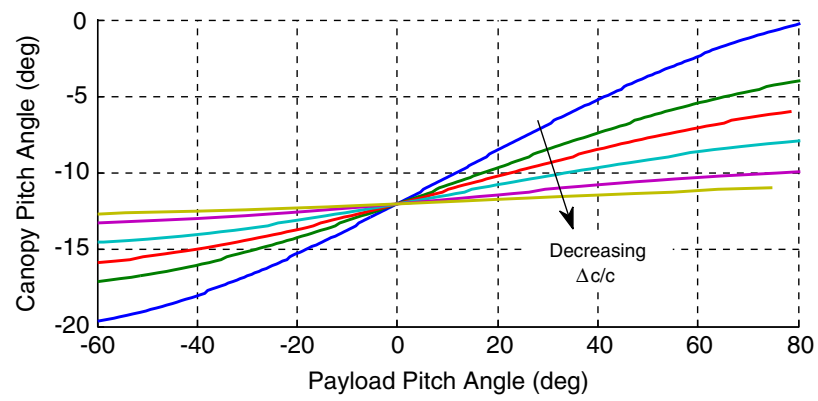

Fig. 15 Canopy pitch angle vs payload pitch angle with separation of rigging attachments.

primary effect of the separation of the rigging attachments is clearly the influence on the sensitivity of the canopy pitch angle to changes in payload pitch angle, as shown in Fig. 15. As the rigging attachment points are moved closer together, the canopy pitch angle becomes increasingly insensitive to changes in the payload pitch angle. If there is no longitudinal separation between rigging attachments, the canopy pitch angle is not influenced by the payload pitch angle and the location of the center of gravity of the payload has no effect on the flight characteristics. In fact, parafoil canopies are commonly rigged in this manner to ensure that the system maintains the design canopy pitch angle in spite of any variations in the payload. Because the relationship between payload pitch and canopy pitch is highly sensitive to the rigging separation, increasing the separation distance produces a large increase in both the sensitivity of canopy pitch angle to weight shift and the range of control over canopy pitch angle. This implies that increasing the separation of the rigging attachments is the primary method of increasing the effectiveness of the longitudinal weight shift control mechanism. The pitch angle vs weight shift behavior for the rigid-body representation of the parafoil-andpayload system is again compared to the multibody results in Fig. 13 to emphasize that the multibody representation is essential to understanding the weight shift control mechanism.

Simulation results from the dynamic multibody model of the X-38 parafoil and payload are shown in Fig. 16. The c.g. is shifted forward from the nominal position by a distance equivalent to $10 \%$ of the canopy chord. The c.g. begins to move at $t=5 \mathrm{~s}$ and takes $2 \mathrm{~s}$ to reach the final value. The vertical distance is $10 \%$ of the rigging line length, and the rigging attachment separation is $10 \%$ of the chord length. The c.g. shift causes the payload to pitch down $35 \mathrm{deg}$ with a resulting change in canopy pitch from the nominal value of $-12 \mathrm{deg}$ down to $-15.5 \mathrm{deg}$.

In the first simulation case, the rigging attachment points on the canopy and payload are constrained in translation but unconstrained in rotation. When the c.g. is moved, two oscillations are excited. There is an oscillation primarily of the payload with a period of approximately $2.5 \mathrm{~s}$ and a second oscillation primarily of the canopy with a period of approximately $13 \mathrm{~s}$. The slower oscillation of the canopy is a large-scale motion caused by a combination of the pendulumlike stability of the parafoil-and-payload system and an oscillating exchange of airspeed and altitude as the canopy settles on
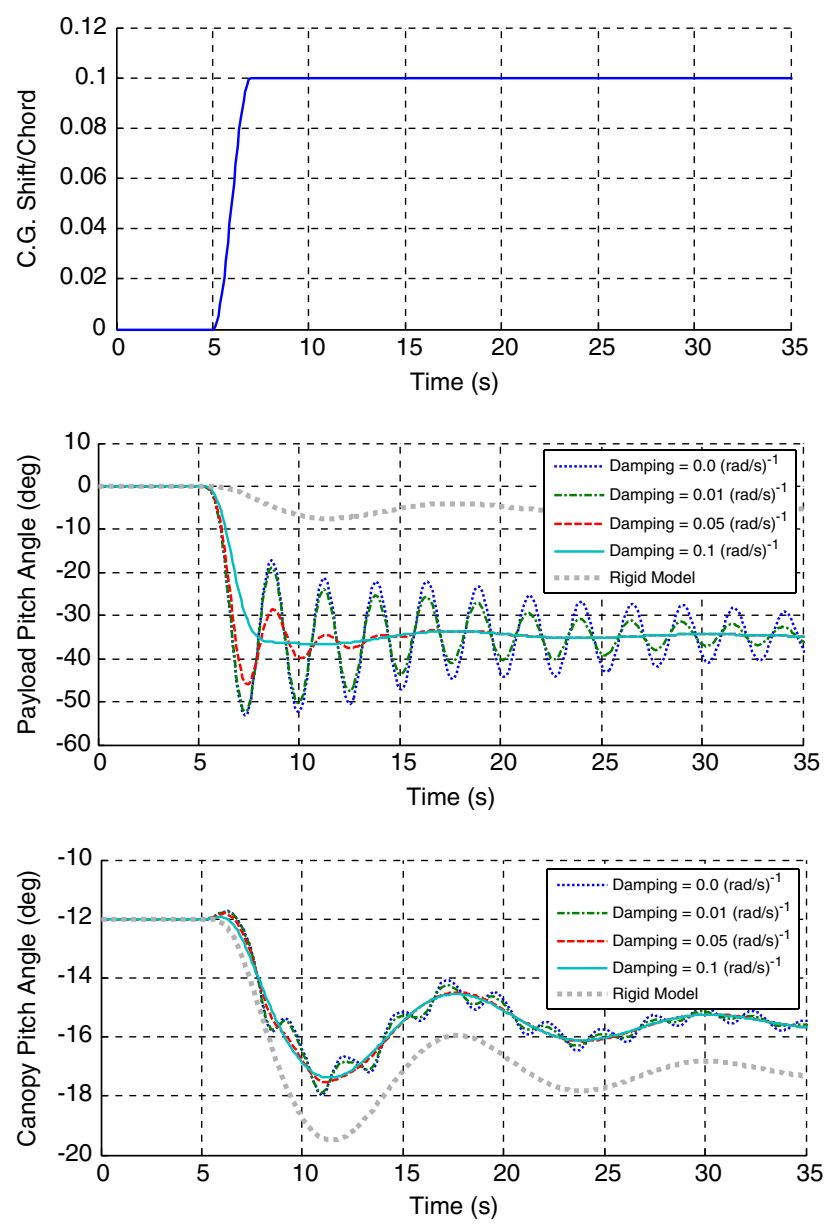

Fig. 16 Dynamic response to weight shift with varying damping in the connections.

its new flight path. This longer period oscillation is the parafoil-andpayload equivalent of the phugoid mode observed with conventional aircraft. The faster oscillation of the payload is a result of the four-bar linkage dynamics and is very lightly damped because the rigging attachment points are allowed to rotate freely. In reality, some damping would be associated with the rotation at the rigging attachments. To simulate this, a damping moment is added proportional
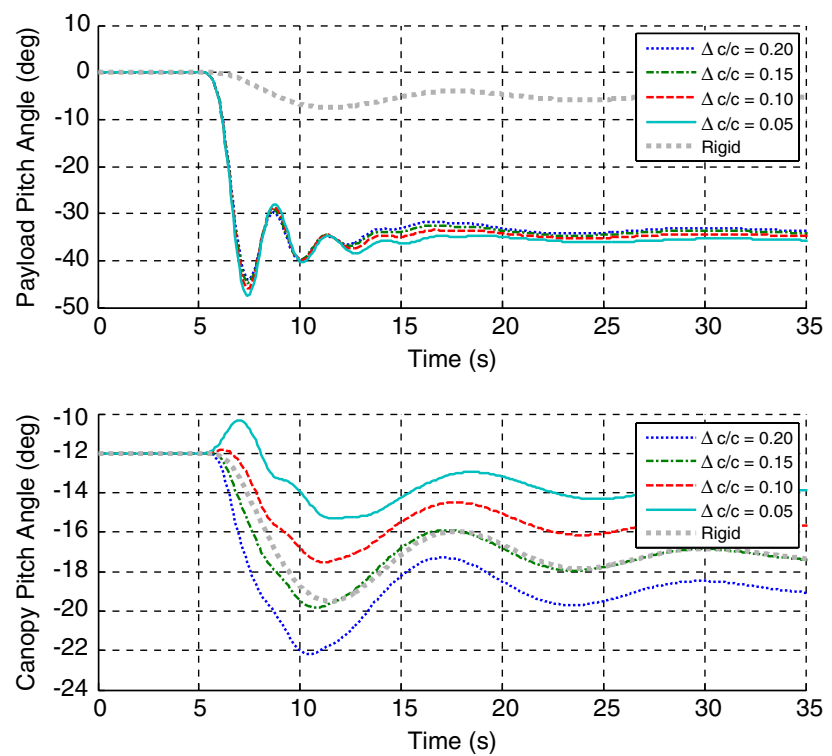

Fig. 17 Dynamic response to weight shift with varying separation of rigging attachments. 
to the relative rotation rate between the bodies at each joint, and the damping constant is normalized by the product of the payload weight and canopy chord. The simulation was repeated with normalized damping constants of $0.001,0.01$, and $0.05(\mathrm{rad} / \mathrm{s})^{-1}$. Note that the damping reduces the oscillation of the payload but has no effect on the longer-scale oscillation of the canopy.

Simulation results from the dynamic multibody model of the X-38 parafoil and payload with varying separation of the rigging attachment points ( $\Delta c$ in Fig. 2) are shown in Fig. 17. The c.g. input is the same as that shown in Fig. $\underline{16}$. For these simulations, the vertical distance is $10 \%$ of the rigging line length, and the normalized damping constant for the rigging attachments was set to $0.01(\mathrm{rad} / \mathrm{s})^{-1}$. The response of the payload is nearly independent of the separation of the rigging attachment points, and while the steadystate canopy pitch angle is altered by the separation of the rigging attachments, the frequency and damping of the oscillations of the canopy are governed by the canopy aerodynamics. However, significant forces and moments are required to actually shift the center of gravity. For example, when the center of gravity is shifted forward to induce a steady-state nosedown pitch to the canopy, the initial moments required to shift the c.g. tend to pitch the canopy nose up. This tendency is increased as the separation of the attachment points is reduced.

Simulation results from the dynamic multibody model with varying heights from the center of gravity to the rigging attachments ( $\Delta h$ in Fig. 2) are shown in Fig. 18. The c.g. input has the same shape as the input shown in Fig. 16; however, the end point of the c.g. shift is adjusted so that the final value of canopy pitch angle is $-16 \mathrm{deg}$ for every case. Because the variation in the rigging attachment height does not affect the relationship between the canopy and payload pitch angles, the steady-state canopy and pitch angle values are the same for each case. The frequencies of the payload oscillations excited by the weight shift are actually increased as the heights of the rigging attachments are increased. Furthermore, the effect of these oscilla-
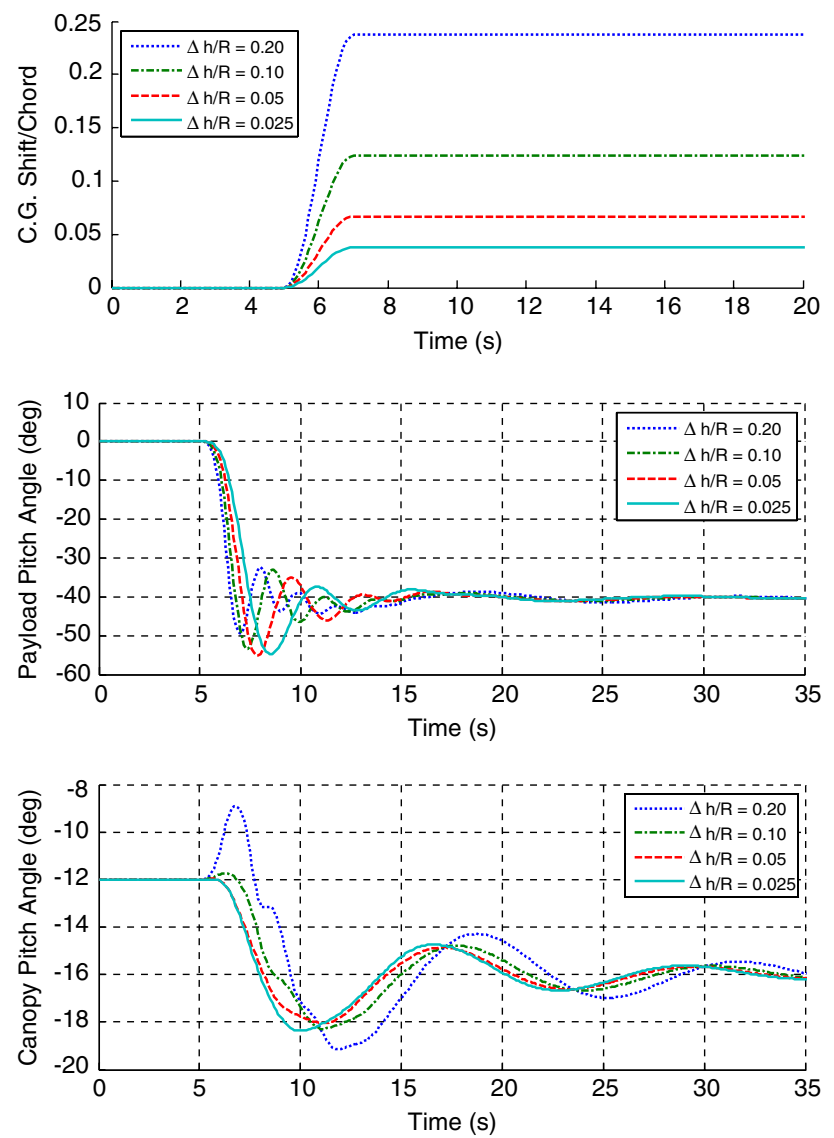

Fig. 18 Dynamic response to weight shift with varying heights of rigging attachments. tions on the canopy pitch angle is amplified as the height of the rigging attachments is increased.

A comparison of the dynamic responses to longitudinal weight shift for the two example systems is given in Fig. 19. The longitudinal separation of the attachment points is $20 \%$ of the canopy chord, the vertical height from the center of gravity to the rigging attachments is $5 \%$ of the average rigging line length, and the payload is shifted forward $15 \%$ of the canopy chord over a period of $2 \mathrm{~s}$ starting at $t=5 \mathrm{~s}$. This weight shift produces a $60 \mathrm{deg}$ nosedown pitch of the payload and a $10 \mathrm{deg}$ nosedown pitch of the canopy for both systems. Referring to Fig. 7, this is enough to produce approximately a $20 \%$ increase in airspeed and a 35\% reduction in glide ratio for both systems. Typical parafoil-and-payload systems used for cargo delivery have no means of glide slope control, but the GT-Imp flighttesting program demonstrated the same range of canopy pitch trim control by varying rigging geometry in flight [27].

While the scalability of longitudinal weight shift to large systems like the X-38 is simple to simulate, it would be very difficult to implement in practice. For the GT-Imp, the $15 \%$ forward weight shift translates to a dimensional distance of roughly $5 \mathrm{in}$. $(0.13 \mathrm{~m})$, but for the $\mathrm{X}-38$, the $15 \%$ forward weight shift translates to a dimensional distance of nearly $7 \mathrm{ft}(2.1 \mathrm{~m})$. While theoretically possible, the actuators and mechanisms required to shift the relative position of the attachment points and the 13,000 lb payload by $7 \mathrm{ft}$ in a few seconds would be extremely expensive and heavy. This is the primary reason why weight shift control is more promising for smaller systems, where actuator weight and cost are less of a concern.

\section{Lateral Weight Shift}

The purpose of lateral weight shift is to induce a turn rate. A lateral shift of the center of gravity relative to the aerodynamic center induces a bank angle. This results in a side slip that, in turn, produces roll and yaw moments on the canopy. The result is a steady turn in the direction of the weight shift. A visualization of the effect of lateral weight shift is shown in Fig. 20.

The direct effect of lateral weight shift is to induce a bank angle in the payload, which is then transmitted to the canopy. The aerodynamics of the canopy determine how that bank angle translates into a turn rate. Figure 21 shows the relationship between canopy bank angle and turn rate for the two example systems. For context, the turn rate limits used for autonomous flight of the example systems are also shown. These turn rate limits are set to ensure that the system does not
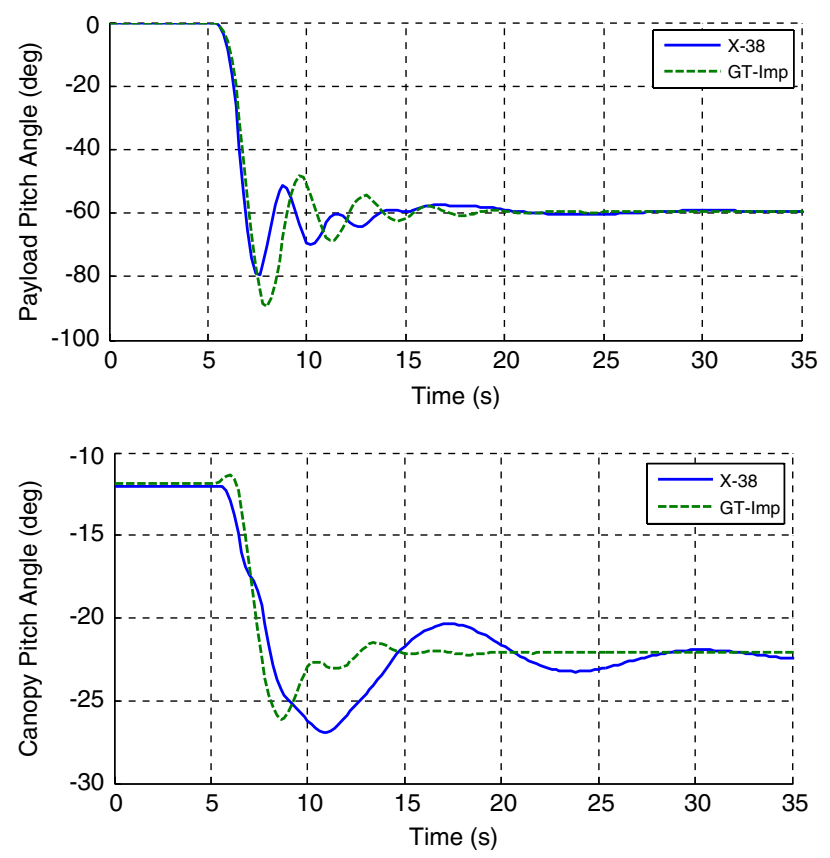

Fig. 19 Comparing dynamic response to weight shift for the two example systems. 

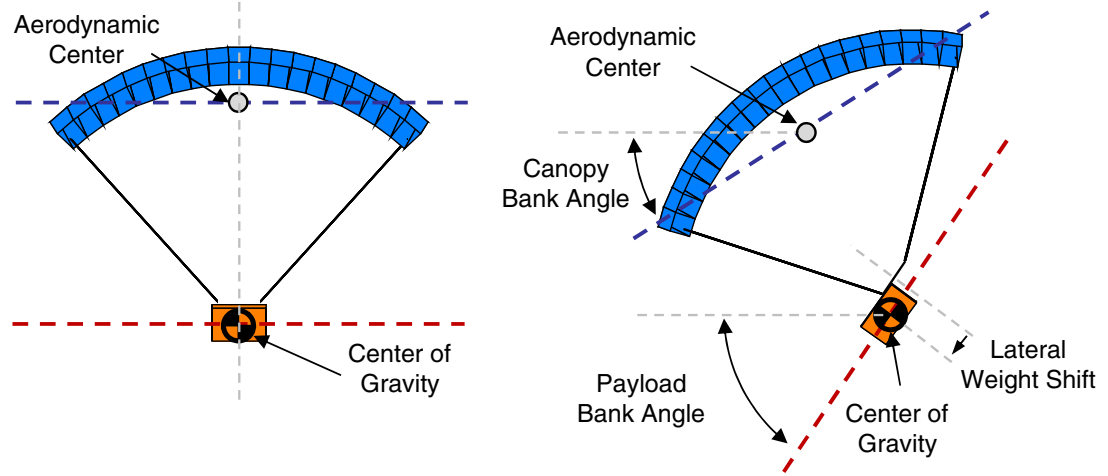

Fig. 20 Visualization of lateral weight shift.

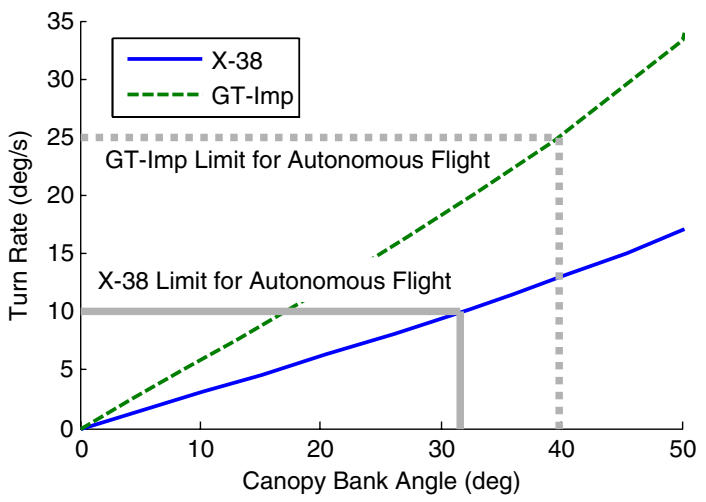

Fig. 21 Relationship between canopy bank angle and turn rate for example systems.

enter a spiral dive during autonomous flight [31], and these limits can be used as control authority targets for evaluating the effectiveness of the lateral weight shift control mechanism.

Figures 22 and 23 show the steady-state canopy and payload bank angles resulting from lateral weight shift with varying separation between the rigging attachment points. Results are shown for the both
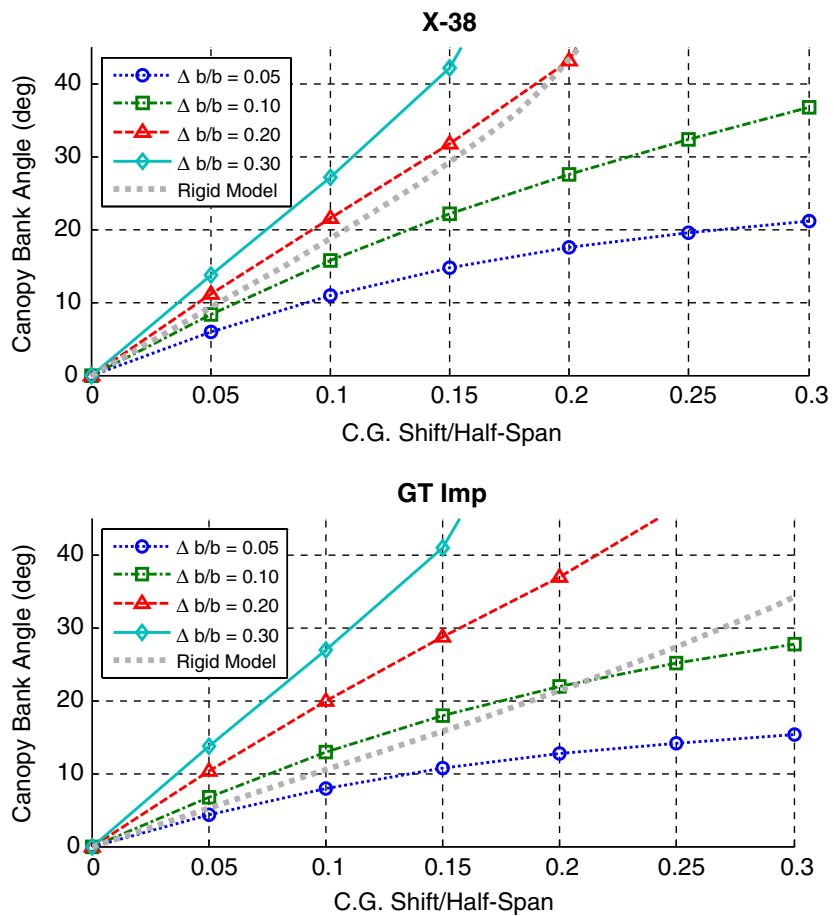

Fig. 22 Canopy bank angle vs lateral weight shift with varying lateral separation of rigging attachments. the X-38 and the GT-Imp. The interaction of the side slip, roll, and yaw dynamics during a turn makes it impossible to reduce the lateral response to weight shift to a single set of nondimensional relationships as was done in the longitudinal case. However, note that, while the steady-state responses of the two systems are slightly different, the trends in the effects of the rigging geometry on the lateral control effectiveness are the same.

The separation of the rigging attachments is normalized by the wingspan, and the lateral weight shift is normalized by half of the wingspan. Similar to the longitudinal case, the separation of the rigging attachments has a very small effect on the relationship between weight shift and payload bank angle until the system approaches the transition to a spiral turn. Increasing the separation between the attachments causes a larger canopy bank angle to result from the same payload bank angle. The response to weight shift using a completely rigid representation of the parafoil and payload is also shown. As with the longitudinal case, the response predicted by the rigid model is similar to the response of the multibody model. However, the effect of weight shift is nonlinear in both cases. For the rigid model, the canopy bank angle actually becomes increasingly sensitive to weight shift as weight shift is increased, while the opposite trend is observed for multibody models with small amounts of separation of the rigging attachments. This again reinforces the

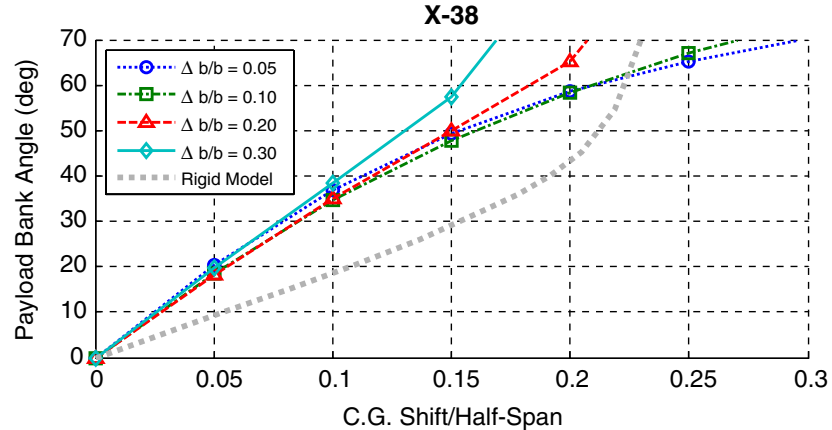

GT Imp

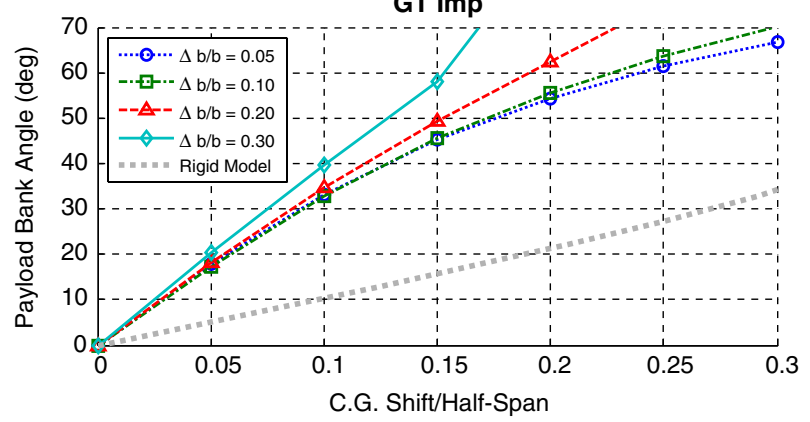

Fig. 23 Payload bank angle vs lateral weight shift with varying lateral separation of rigging attachments. 
need for a multibody simulation approach to gain insight into the weight shift control mechanism.

Figure 24 shows the canopy and payload bank angles that result from lateral weight shift while varying the height of the rigging attachments from the center of gravity. The lateral separation of the attachments was $10 \%$ of the canopy span for these cases. Similar to the longitudinal case, the sensitivity to lateral weight shift is increased as the vertical distance from the center of gravity to the rigging attachments is decreased. Again, the same trends are observed for the $\mathrm{X}-38$ and the GT-Imp models.

Figure 25 shows the relationship between payload bank angle and canopy bank angle. Similar to the longitudinal case, the vertical distance between the rigging attachments and the center of gravity does not influence this relationship. However, unlike the longitudinal case, the differences in the lateral aerodynamic characteristics of the X-38 and GT-Imp canopies result in a slightly different relationship between the payload and canopy bank angles.

The next set of plots examines the dynamic response to lateral weight shift. Figure 26 shows the input used for the simulations. The center of gravity is moved from the center to a point $10 \%$ of the half-span to the right. The weight shift starts at $5 \mathrm{~s}$ and takes $2 \mathrm{~s}$ to complete. The GT-Imp model is used for all of these dynamic simulations.
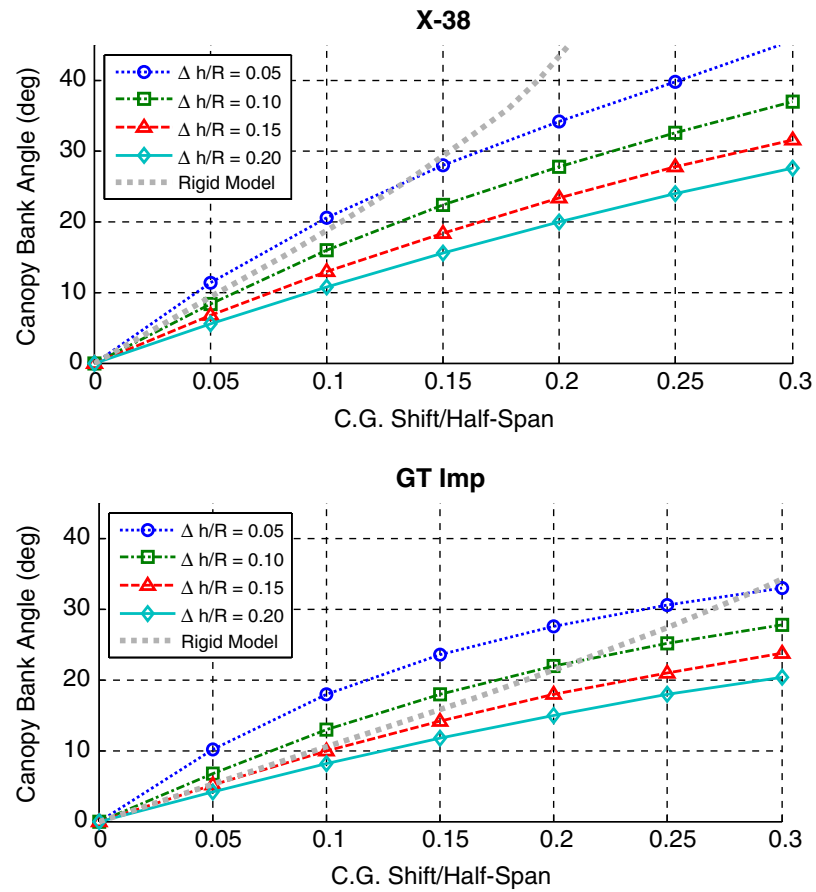

Fig. 24 Canopy bank angle vs lateral weight shift with varying heights of rigging attachments above center of gravity.

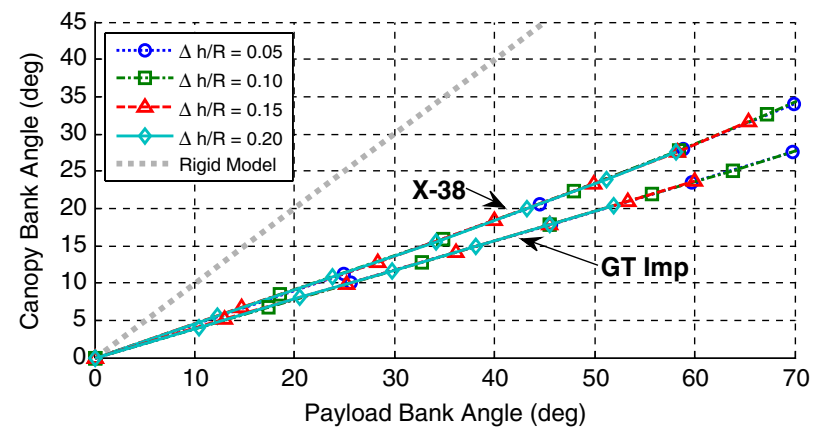

Fig. 25 Canopy bank angle vs payload bank angle with varying heights of rigging attachments above center of gravity.

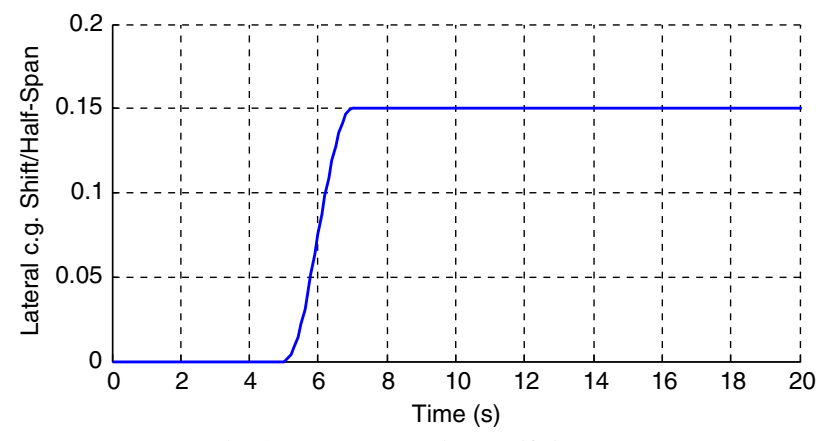

Fig. 26 Lateral weight shift input.

The payload bank angle, canopy bank angle, and turn rate responses to the lateral weight shift are shown for varying values of the attachment point separation in Fig. 27. The response of the completely rigid simulation model is also shown. Similar to the longitudinal case, the dynamic response of the canopy to the weight shift is composed of two parts. There is a short-period oscillation associated with the relative motion of the payload and canopy, and there is a slower response associated with the rigid-body dynamics that are governed by the canopy aerodynamics. As the separation distance between the attachment points is decreased, the damping of the short-period oscillation of the payload is decreased. This oscillation feeds back into both the canopy bank angle and turn rate response. Decreasing the separation distance also decreases the magnitude of the response to the weight shift input, although the time to settle to the steady-state response does not appear to be strongly
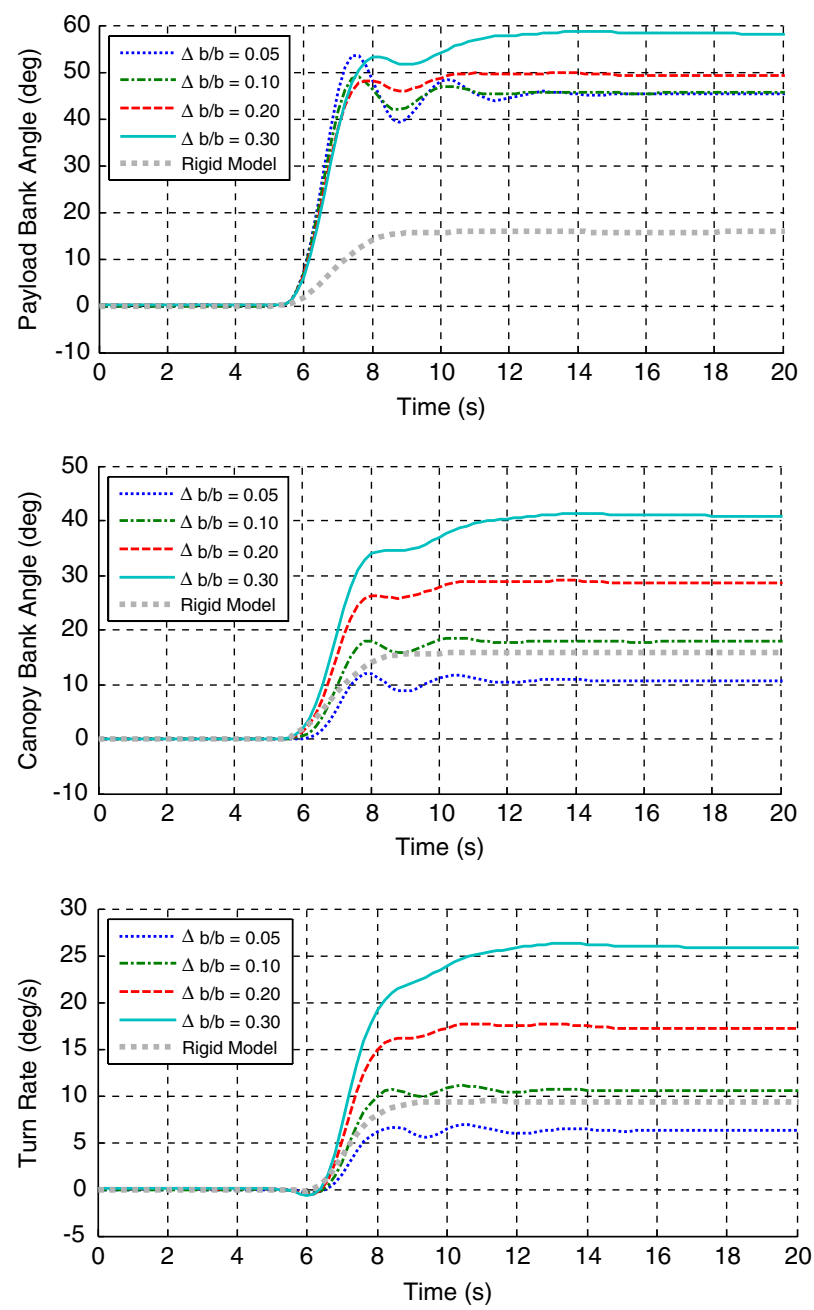

Fig. 27 Dynamic response to lateral weight shift with varying lateral separation of rigging attachments. 

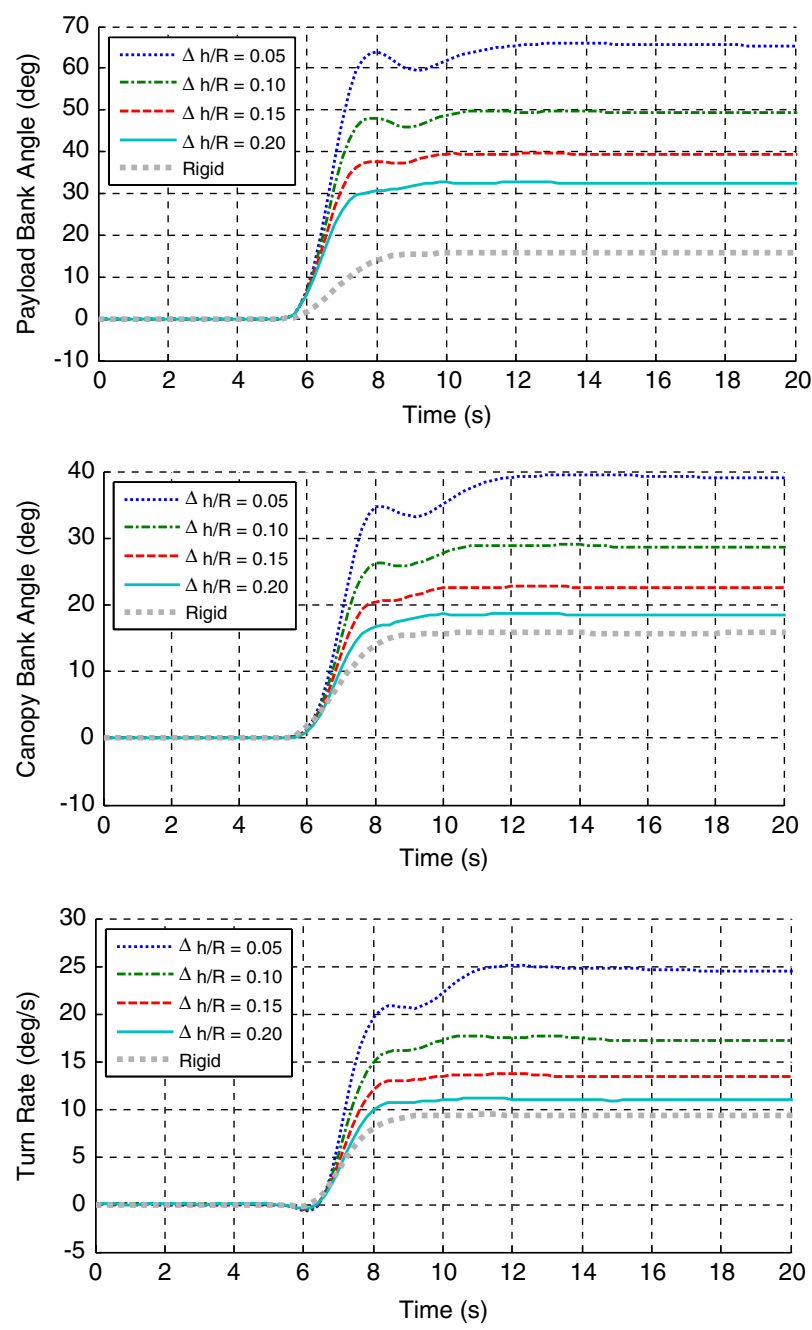

Fig. 28 Dynamic response to lateral weight shift with varying heights of rigging attachments above center of gravity.

influenced by the attachment geometry, indicating that the settling time is dominated by the canopy aerodynamics. The initial turn rate response is in the opposite direction to the weight shift. This effect is a response to the initial forces and moments required to shift the center of gravity from its nominal position, and it is present for the rigid model as well as the multibody models.

The payload bank angle, canopy bank angle, and turn rate responses to the lateral weight shift input in Fig. 26 are shown with varying values of the height of the attachment points from the center of gravity in Fig. 28. The separation of the attachment points was $20 \%$ of the span for all of these simulations. Similar to the lateral separation of the rigging, the vertical distance between the rigging attachments and the payload c.g. affects the damping of the payload oscillations as well as the magnitudes of the steady-state bank angles and turn rate. As the distance is decreased, the oscillation becomes more apparent. This oscillation feeds back into both the canopy bank angle and turn rate response.

The dynamic responses to lateral weight shift for the X-38 and GTImp models are shown in Fig. 29. The lateral separation of the rigging attachments was $20 \%$ of the canopy span, and the vertical distance from the center of gravity to the rigging attachments was $5 \%$ of the rigging line length for both cases. The magnitude of the lateral weight shift was selected to achieve the steady-state turn rate limit imposed on each system during autonomous operations. For the X-38, a lateral weight shift of $7 \mathrm{ft}(2.1 \mathrm{~m}, 11 \%$ of the half-span $)$ is required to achieve a $10 \mathrm{deg} / \mathrm{s}$ turn rate. For the GT-Imp, a lateral weight shift of 7 in. $(0.18 \mathrm{~m}, 15 \%$ of the half-span $)$ is required to achieve a $25 \mathrm{deg} / \mathrm{s}$ turn rate. These results again indicate that weight shift as a control mechanism is more practical for smaller-scale systems.
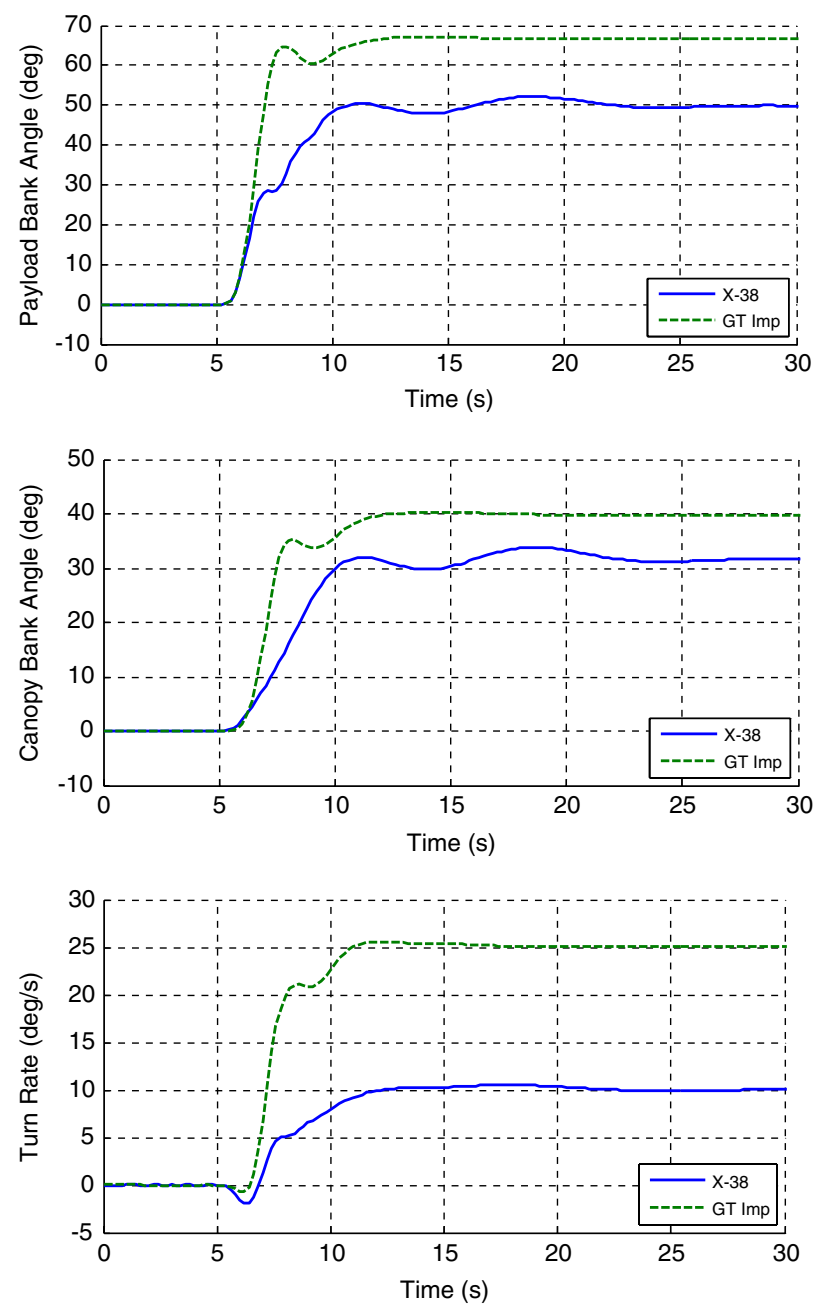

Fig. 29 Dynamic response to lateral weight shift for the two example systems.

\section{Conclusions}

Multibody simulation results demonstrate that weight shift control is an effective means of providing longitudinal and lateral control of parafoil-and-payload aircraft. The results of these simulations were also compared with a rigid representation to demonstrate that the multibody approach is critical to understanding the effect of weight shift on this type of aircraft. Results demonstrate that longitudinal weight shift can be used to provide effective control of airspeed and glide ratio by altering the trim canopy pitch angle, and lateral weight shift can be used to provide effective turn rate control. The geometry of the attachments of the rigging to the payload strongly influences the effectiveness of payload weight shift as a control mechanism. Results demonstrate that, for both lateral and longitudinal control, spreading the attachment points of the rigging to the payload will cause the aircraft to be more sensitive to shifts in the payload center of gravity. It was also found that, for both lateral and longitudinal control, decreasing the vertical distance from the rigging attachments to the center of gravity on the payload causes the aircraft to be more sensitive to shifts in the payload center of gravity. Dynamic results demonstrate that the forces and moments required to induce a weight shift in the payload will often cause an initial response in the opposite direction of the final steady-state response.

\section{Acknowledgments}

This work was supported by the U.S. Army Natick Soldier Research, Development and Engineering Center, with Steve Tavan serving as the Contract Monitor. 


\section{References}

[1] Murray, J., Sim, A., Neufeld, D., Rennich, P., Norris, S., and Hughes, W., "Further Development and Flight Test of an Autonomous Precision Landing System Using a Parafoil," NASA TM-4599, July 1994.

[2] Kaminer, I., and Yakimenko, O., "Development of Control Algorithm for the Autonomous Gliding Delivery System," AIAA Paper 20032116, May 2003.

[3] Jann, T., "Advanced Features for Autonomous Parafoil Guidance, Navigation and Control," AIAA Paper 2005-1642, May 2005.

[4] Carter, D., George, S., Hattis, P., Singh, L., and Tavan, S., "Autonomous Guidance, Navigation, and Control of Large Parafoils," AIAA Paper 2005-1643, May 2005.

[5] Carter, D., George, S., Hattis, P., McConley, M., Rasmussen, S., Singh, L., and Tavan, S., "Autonomous Large Parafoil Guidance, Navigation, and Control System Design Status," AIAA Paper 2007-2514, May 2007.

[6] Calise, A., and Preston, D., "Swarming/Flocking and Collision Avoidance for Mass Airdrop of Autonomous Guided Parafoils," Journal of Guidance, Control, and Dynamics, Vol. 31, No. 4, 2008, pp 1123-1132. doi: $10.2514 / 1.28586$

[7] Carter, D., Singh, L., Wholey, L., Rasmussen, S., Barrows, T., George, S., McConley, M., Gibson, C., Tavan, S., and Bagdonovich, B., "Band-Limited Guidance and Control of Large Parafoils," AIAA Paper 2009-2981, May 2009.

[8] Slegers, N., and Yakimenko, O., "Optimal Control for Terminal Guidance of Autonomous Parafoils," AIAA Paper 2009-2958, 2009.

[9] Gratton, G. B., "The Weightshift-Controlled Microlight Aero-Plane," Journal of Aerospace Engineering, Vol. 214, No. 93, 2001, pp. 147-154.

[10] Brown, G., "New Trends and Developments in Hang Gliders," 2nd MIT and SSA International Symposium on the Technology and Science of Low Speed and Motorless Flight, AIAA Paper 74-1031, Sept. 1974.

[11] Anderson, S., and Ormiston, R., "A Look at Handling Qualities of High Performance Hang Gliders," AIAA Atmospheric Flight Mechanics Conference, AIAA Paper 1994-3492, 1994.

[12] Dees, P., "Hang Glider Design and Performance," 10th AIAA Aviation Technology, Integration, and Operations (ATIO) Conference, AIAA Paper 2010-9300, Sept.. 2010.

[13] Weight-Shift Control Aircraft Flying Handbook, U.S. Dept. of Transportation, Federal Aviation Administration, Flight Standards Service, FAA-H-8083-5, 2008.

[14] Whittall, N., Paragliding: The Complete Guide, Lyons Press, New York, 1995, Chaps. 2, 7.

[15] Barrows, T., "Apparent Mass of Parafoils with Spanwise Camber," Journal of Aircraft, Vol. 39, No. 3, 2002, pp. 445-451. doi: $10.2514 / 2.2949$

[16] Slegers, N., Beyer, E., and Costello, M., "Use of Variable Incidence Angle for Glide Slope Control of Autonomous Parafoils," Journal of Guidance Control and Dynamics, Vol. 31, No. 3, 2008, pp 585-596. doi: $10.2514 / 1.32099$
[17] Mortaloni, P., Yakimenko, O., Dobrokhodov, V., and Howard, R., "On the Development of a Six-Degree-of-Freedom Model of a LowAspect-Ratio Parafoil Delivery System," 17th AIAA Aerodynamic Decelerator Systems Technology Conference and Seminar, AIAA Paper 2003-2105, 2003.

[18] Mooij, E., Wijnands, Q., and Schat, B., "9-DOF Parafoil/Payload Simulator Development and Validation," AIAA Paper 2003-5459, Aug. 2003.

[19] Müller, S., Wagner, O., and Sachs, G., "A High-Fidelity Nonlinear Multibody Simulation Model for Parafoil Systems," AIAA Paper 2003-2120, May 2003.

[20] Redelinghuys, R., "A Flight Simulation Algorithm for a Parafoil Suspending an Air Vehicle," Journal of Guidance, Control, and Dynamics, Vol. 30, No. 3, 2007, pp. 791-803. doi: $10.2514 / 1.25074$

[21] Slegers, N., "Effects of Canopy-Payload Relative Motion on Control of Autonomous Parafoils," Journal of Guidance, Control, and Dynamics, Vol. 33, No. 1, 2010, pp. 116-125. doi: $10.2514 / 1.44564$

[22] Wise, K., "Dynamics of a UAV with Parafoil Under Powered Flight," AIAA Guidance, Navigation, and Control Conference and Exhibit, AIAA Paper 2006-6795, 2006.

[23] Strickert, G., and Witte, L., "Analysis of the Relative Motion in a Parafoil-Load-System," AIAA Paper 2001-2013, 2001.

[24] Costello, M., French, K., and Leylek, E., "Flight Dynamic Simulation for Multibody Aircraft Configurations," AIAA Modeling and Simulation Technologies Conference, AIAA Paper 2010-2013, 2010.

[25] Leylek, E., Ward, M., and Costello, M., "Flight Dynamic Simulation for Multibody Aircraft Configurations," Journal of Guidance, Control, and Dynamics, Vol. 35, No. 6, 2012, pp. 1828-1842. doi: $10.2514 / 1.55858$

[26] Coleman, T. F., and Li, Y., "An Interior, Trust Region Approach for Nonlinear Minimization Subject to Bounds," SIAM Journal on Optimization, Vol. 6, No. 2, 1996, pp. 418-445. doi: $10.1137 / 0806023$

[27] Ward, M., Gavrilovski, A., and Costello, M., "Flight Test Results for Glide Slope Control of Parafoil Canopies of Various Aspect Ratios," AIAA Paper 2011-2620, 2011.

[28] Iacomini, C. S., and Cerimele, C. J. "Longitudinal Aerodynamics From a Large Scale Parafoil Test Program,” AIAA Paper 1999-1732, 1999.

[29] Jann, T., "Aerodynamic Coefficients for a Parafoil Wing with Arc Anhedral: Theoretical and Experimental Results," 17th AIAA Aerodynamic Decelerator Systems Technology Conference and Seminar, AIAA Paper 2003-2106, May 2003.

[30] Lingard, J. S., "Precision Aerial Delivery Seminar: Ram-Air Parachute Design," 13th AIAA Aerodynamic Decelerator Systems Technology Seminar, AIAA Paper, 1995.

[31] Iacomini, C., and Cerimele, C., "Lateral-Directional Aerodynamics from a Large Scale Parafoil Test Program," AIAA Paper 1999-1731, 1999. 